\title{
Pulsed-Field Gel Electrophoresis of Genomic Digests of Thermus Strains and Its Implications for Taxonomic and Evolutionary Studies
}

\author{
A. G. RODRIGO, ${ }^{1}$ K. M. BORGES, ${ }^{2}$ AND P. L. BERGQUIST ${ }^{3 *}$ \\ Experimental Biology Research Group, School of Biological Sciences, ${ }^{1}$ and Centre for Gene Technology, ${ }^{3}$ University of \\ Auckland, Auckland, New Zealand, and Center for Marine Biotechnology, Baltimore, Maryland $21202^{2}$
}

\begin{abstract}
Pulsed-field electrophoresis (PFGE) of the SspI genomic digests of 14 Thermus isolates showed that each one had a unique restriction enzyme digestion pattern. A group of New Zealand strains showed some shared bands, but each isolate gave essentially a unique fingerprint. In addition, evolutionary distances between Thermus strains estimated by using PFGE restriction fragment length polymorphisms (PFGE-RFLPs) correlate well with those based on small-subunit rRNA sequence data. As a consequence, the phylogenetic trees constructed on the basis of PFGE-RFLPs and those constructed by using small-subunit rRNA sequences generally agree. On the basis of the evolutionary distances estimated by using PFGE-RFLPs, the estimated average genomic rate of divergence for Thermus spp. is approximately $0.27 \%$ per million years.
\end{abstract}

Many strains of thermophilic bacteria isolated from hot springs located around the world have been classified as belonging to the genus Thermus, but the relationships among these strains have not been well established. Only three species of Thermus have been validly described so far $(3,11,15)$ : Thermus aquaticus, originally isolated from hot springs in Yellowstone National Park; Thermus filiformis, a single strain isolated in New Zealand; and Thermus ruber, isolated from many locations, and distinguished from other Thermus strains by red pigmentation and a lower growth temperature. "Thermus thermophilus" (20) is thought to be a separate species, though it has not been formally accepted. Several Thermus isolates show high DNA-DNA homology with HB8, the " $T$. thermophilus" type strain (28). Whether all the Thermus isolates obtained are taxonomic variants of the three nominal species or are species themselves is the source of some debate.

A number of studies have attempted to resolve the status of Thermus strains. Phenetic studies have been performed to classify Thermus isolates from New Zealand (10), Iceland (4), and Portugal (22) on the basis of microbiological and phenotypic characters. In addition, a phenetic classification of 131 Thermus strains isolated from hot springs around the world has been performed (12). On the basis of analysis of 68 phenotypic characteristics, eight distinct groups of Thermus strains were defined at the level of similarity separating the three recognized species. Classifications based on numerical taxonomy, however, often fail to identify groups that share close evolutionary relationships, and it is precisely these relationships that many systematists feel should form the basis of any classification.

Therefore, another approach to resolving the problems with the classification of the Thermus strains is to use procedures explicitly designed to uncover the evolutionary relationships or phylogeny of the taxa in question. In a recent paper, Saul et al. (23) used small-subunit (SSU) rRNA sequences of 20 Thermus strains to reconstruct their phylogenetic history. The study showed that of the 20 strains, there were four primary clades

\footnotetext{
* Corresponding author. Mailing address: Centre for Gene Technology, University of Auckland, Private Bag 92019, Auckland, New Zealand. Phone: 64-9-3737599, ext. 7712. Fax: 64-9-3737414.
}

that were separated from each other at a statistically significant level.

While molecular sequences often provide reliable estimates of phylogeny, there are other more accessible sources of information that are as reliable, depending, of course, on the extent to which the taxa are evolutionarily divergent (8). Recently, several workers have used pulsed-field gel electrophoresis (PFGE) to compare genomic restriction digests amongst bacterial strains. This method is equivalent to the identification of restriction fragment length polymorphisms (RFLPs) and can allow a unique DNA fingerprint to be obtained for a bacterial isolate. PFGE-RFLPs have been used successfully to distinguish among isolates of several bacteria, including Listeria species (9), Lactococcus strains (14, 27), and Pseudomonas aeruginosa strains (7). To date, however, no one has reported on the possibility of using this method for phylogenetic reconstruction and classification.

In this paper, we describe the comparison and phylogenetic analysis of PFGE fingerprints from 14 Thermus strains. We show that (i) PFGE-RFLPs can successfully discriminate between different strains of Thermus; (ii) the evolutionary distances between Thermus strains estimated by using PFGERFLP data are significantly correlated with those obtained from SSU rRNA sequences; (iii) PFGE-RFLPs produce phylogenetic trees that are independently corroborated by SSU rRNA data; and finally, (iv) on the basis of the evolutionary distances estimated from PFGE-RFLPs, we can obtain an estimate of the average rate of nucleotide substitution across the entire Thermus genome of 0.18 to 0.36 nucleotide substitutions per site per million years.

\section{MATERIALS AND METHODS}

Fingerprinting of Thermus strains bv PFGE. Intact chromosomal DNA in agarose gel plugs was prepared from 14 Thermus strains previously isolated from hot springs in Japan, the United States, New Zealand, and Fiji: "T. thermophilus" HB8, "T. thermophilus" HB27, T. aquaticus YT-1, T. aquaticus YVII-51B, Thermus sp. strain Rt41A, Thermus sp. strain OK6, Thermus sp. strain T. sp., Thermus sp. strain T351, Thermus sp. strain W28, Thermus sp. strain W28P, Thermus sp. strain Tok3, Thermus sp. strain Tok20, Thermus sp. strain Tok21, and Thermus sp. strain Fiji3. DNA was prepared as previously 
described (1) either by the Thermus Rt41A proteinase method modified by use of $45 \mathrm{U}$ of proteinase per 6 gel plugs (2), or by the standard proteinase $\mathrm{K}$ method of Smith et al. (24).

The genome of " $T$. thermophilus" HB8 has been mapped previously by PFGE techniques (2), and during map construction, several restriction endonucleases were screened for infrequent cleavage of the $69 \% \mathrm{G}+\mathrm{C}$ genome. Since the endonuclease HpaI generated only six fragments with the " $T$. thermophilus" HB8 genome, it was tested in this study for rare cleavage of other Thermus genomes. However, most of the Thermus chromosomes were cleaved by $\mathrm{HpaI}$ into too many fragments of similar sizes to be adequately resolved by PFGE (data not shown) and after further screening the enzyme SspI was chosen for this study.

One DNA gel plug was equilibrated with $200 \mu$ l of $S s p$ I buffer (Gibco BRL/Life Technologies, Auckland, New Zealand) at $4^{\circ} \mathrm{C}$ for $20 \mathrm{~min}$. The buffer was replaced, and DNA was digested with $20 \mathrm{U}$ of $S s p \mathrm{I}$ for $16 \mathrm{~h}$ in a $100-\mu \mathrm{l}$ reaction volume. After digestion, buffer was removed and each gel plug was equilibrated with $1 \mathrm{ml}$ of Tris-EDTA buffer at $4^{\circ} \mathrm{C}$ for at least $20 \mathrm{~min}$ prior to electrophoresis.

One-fifth of each of the gel plugs was loaded onto a $1 \%$ agarose MP (Boehringer-Mannheim, Auckland, New Zealand) gel, and PFGE was performed in $0.5 \times$ TBE buffer $(0.045 \mathrm{M}$ Tris-borate, $0.001 \mathrm{M}$ EDTA [pH 8.0]) at $14^{\circ} \mathrm{C}$ with a Bio-Rad Chef DR-II system. Lambda DNA oligomers (FMC BioProducts, Rockland, Maine) were used as a size standard. Two sets of electrophoretic conditions were sufficient to separate all of the SspI fragments for each strain. Most of the fragments were resolved with a pulse time of $20 \mathrm{~s}$ for $24 \mathrm{~h}$ (Fig. 1A), but fragments larger than $390 \mathrm{~kb}$ were separated with a ramped pulse time of 40 to $80 \mathrm{~s}$ for $24 \mathrm{~h}$ (Fig. 1B). Fragment sizes were measured by comparison with the $\lambda$ ladder. Band sizes were analyzed by using a digitizer linked to a Macintosh computer running a software program using interpolation of band sizes based on a cubic spline formula. The program did not generate a straight-line relationship over the entire size range of the $\lambda$ markers. Bands were sized only in the linear portion of the calibration curve, and the pulse time was altered to shift different bands into the linear range.

Phylogenetic analyses. Although it has been customary to perform phylogenetic analyses on restriction fragment or fingerprint profiles using the presence or absence of bands as characters (for an example, see reference 6), this procedure is fraught with problems due, in large part, to the nonindependence of fragments (26). We have used an alternative approach to estimate the evolutionary distance (expressed as the rate of nucleotide substitutions per site) between pairs of taxa from the patterns of band sharing. Nei and $\mathrm{Li}(18)$ showed that for two genomes of comparable sizes, the expected proportion of shared fragments is given by: $F \approx G^{4} /(3-2 G)$, where $G=$ $e^{-s r t}$, and $s$ is the number of bases recognized by the restriction enzyme, $r$ is the rate of nucleotide substitution per site, and $t$ is the time of divergence. Given the RFLP profiles of any two taxa, $x$ and $y$, we can estimate $F$ using the following equation: $\hat{F}=2 n_{x y} / n_{x}+n_{y}$, where $n_{x}$ and $n_{y}$ are the numbers of bands in $x$ and $y$, respectively, and $n_{\mathrm{xy}}$ is the number of bands shared by $x$ and $y$. Because the estimation of $F$ relies on the fact that two taxa share at least one band in common, evolutionary distances between Thermus strains with no bands in common cannot be estimated.

Once $F$ has been estimated, $G$ can be calculated by an iterative algorithm (18), such that $G_{i+1}=\left[F\left(3-2 G_{i}\right)\right]^{0.25}$, where $G_{k}$ is the estimate of $G$ at the $k$ th iteration. Let $G_{1}=$ $F^{0.25}$, and choose a convergence criterion, $\varepsilon$, such that if $\mid G_{i}+$ $1-\mathrm{G}_{\mathrm{i}} \mid<\varepsilon$, then $G_{i+l}$ is taken as the best estimate of $G$. In our
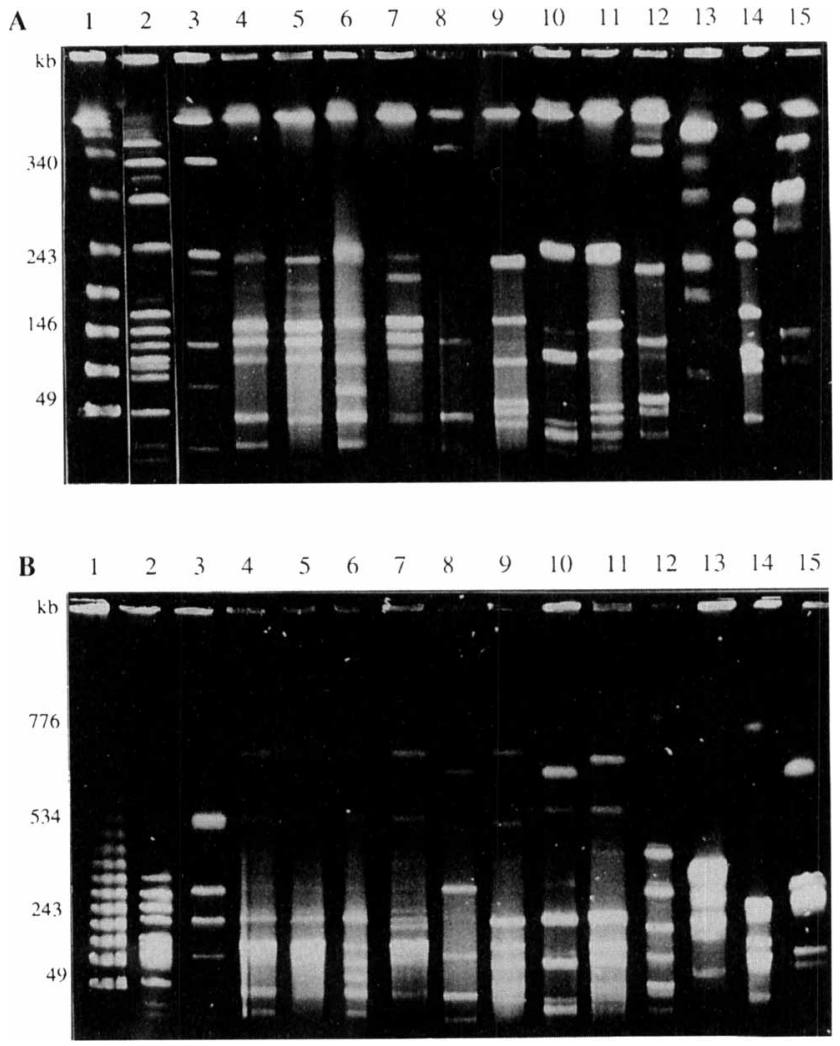

FIG. 1. PFGE analysis of $S s p$ I restriction fragments from Thermus genomic DNA. Samples were prepared and electrophoresed as described in Materials and Methods. (A) PFGE was performed at $200 \mathrm{~V}$ for $24 \mathrm{~h}$ with a pulse time of $20 \mathrm{~s}$. Lanes: 1 , lambda DNA concatemer size standard; 2, T. aquaticus YVII-51B; 3, T. aquaticus YT-1; 4, Thermus sp. strain T351; 5, Thermus sp. strain T. sp.; 6, Thermus sp. strain OK6; 7, Thermus sp. strain Rt41A; 8, Thermus sp. strain W28P; 9, Thermus sp. strain W28; 10, Thermus sp. strain Tok21; 11, Thermus sp. strain Tok $20 ; 12$, Thermus sp. strain Tok3; 13, Thermus sp. strain Fiji3; 14, T. thermophilus HB27; 15, T. thermophilus HB8. (B) PFGE was performed at $200 \mathrm{~V}$ with a ramped pulse time of 40 to $80 \mathrm{~s}$ over 24 h. The lanes are as in panel A.

analysis, $\varepsilon$ was set at 0.0001 . Having obtained $G$ for any two taxa $x$ and $y$, the evolutionary distance between $x$ and $y, d(x, y)$, can be estimated as $d(x, y)=-(2 \ln G) / r$.

The neighbor-joining tree reconstruction method (21) was used to construct a phylogenetic tree of all taxa for which there was a complete set of pairwise evolutionary distances based on PFGE-RFLP data. Neighbor joining has been shown to perform better than other distance-based tree-building methods with simulated data under a range of different models of evolution (17).

\section{RESULTS}

Fingerprinting of Thermus strains by PFGE. The number of bands detected ranged from 6 (for " $T$. thermophilus" HB8 and W28P) to 13 (for $T$. aquaticus YT-1). Table 1 shows the number of bands generated for each strain and their approximate sizes estimated by comparison with a ladder of lambda DNA oligomers. The sum of the restriction fragment sizes for each strain gave an estimate of the genome size. As reported previously, the genome of " $T$. thermophilus" HB8 was determined to be approximately $1.8 \mathrm{Mbp}(2)$. The genomes from 
TABLE 1. Restriction fragments produced by $S s p$ I cleavage of Thermus genomic DNA

\begin{tabular}{|c|c|c|c|c|c|c|c|c|c|c|c|c|c|c|c|}
\hline \multirow{2}{*}{ Strain } & \multirow{2}{*}{$\begin{array}{l}\text { Genome size } \\
\quad(\mathrm{Mbp})\end{array}$} & \multirow{2}{*}{$\begin{array}{c}\text { No. of } \\
\text { SspI bands }\end{array}$} & \multicolumn{13}{|c|}{ Fragment size $(\mathrm{kb})^{a}$} \\
\hline & & & 1 & 2 & 3 & 4 & 5 & 6 & 7 & 8 & 9 & 10 & 11 & 12 & 13 \\
\hline T. thermophilus HB8 & 1.8 & 6 & 101 & 134 & 259 & 290 & 348 & 664 & & & & & & & \\
\hline T. thermophilus HB 27 & 2.0 & 9 & 11 & 37 & 90 & 111 & 160 & 237 & 257 & 275 & 782 & & & & \\
\hline Fiji3 & 2.1 & 8 & 81 & 181 & 221 & 228 & 285 & 317 & 376 & 404 & & & & & \\
\hline Tok3 & 2.1 & 8 & 29 & 44 & 55 & 128 & 221 & 338 & 433 & 841 & & & & & \\
\hline Tok20 & 2.1 & 11 & 16 & 23 & 28 & 38 & 48 & 114 & 149 & 232 & 242 & 561 & 697 & & \\
\hline Tok21 & 2.1 & 11 & 13 & 21 & 27 & 30 & 35 & 109 & 116 & 237 & 243 & 570 & 665 & & \\
\hline W28 & 2.1 & 9 & 34 & 44 & 52 & 103 & 154 & 227 & 234 & 527 & 729 & & & & \\
\hline W28P & 1.3 & 6 & 24 & 42 & 42 & 131 & 344 & 673 & & & & & & & \\
\hline Rt41A & 2.2 & 9 & 17 & 40 & 109 & 131 & 152 & 210 & 234 & 545 & 731 & & & & \\
\hline OK6 & 2.2 & 10 & 18 & 25 & 42 & 67 & 110 & 154 & 237 & 246 & 544 & 719 & & & \\
\hline T. sp. & 1.9 & 8 & 17 & 39 & 110 & 132 & 150 & 234 & 715 & & & & & & \\
\hline T351 & 2.0 & 9 & 17 & 25 & 42 & 110 & 131 & 151 & 235 & 536 & 714 & & & & \\
\hline T. aquaticus YT-1 & 1.5 & 8 & 14 & 23 & 73 & 127 & 218 & 239 & 324 & 519 & & & & & \\
\hline T. aquaticus YVII-51B & 2.0 & 13 & 21 & 29 & 54 & 91 & 101 & 110 & 125 & 142 & 161 & 218 & 257 & 300 & 354 \\
\hline
\end{tabular}

${ }^{a}$ Average of at least two determinations.

most of the other strains were similar in size (measured at 1.9 to $2.1 \mathrm{Mbp}$ ). However, the sums of the fragment sizes for both T. aquaticus YT-1 and Thermus strain W28P were much smaller: 1.5 and $1.3 \mathrm{Mbp}$, respectively. Whether these differences in calculated chromosome size are genuine or are an artifact resulting from incomplete restriction endonuclease digestion or poor separation of bands by PFGE is unknown but could be resolved by comparing calculated genome sizes after cleavage by a variety of rarely cutting endonucleases. All of the Thermus strains tested produced a characteristic and reproducible PFGE band profile. Most of the strains showed quite different banding patterns, with a small number of shared bands. The New Zealand strains Rt41A, T. sp., and T351 showed strikingly similar banding patterns, despite the fact that they were isolated from different locations.

Phylogenetic analysis. Table 2 shows the evolutionary distances between all the taxa examined. Note that for some pairs of strains, no distance has been estimated since the strains in question share no bands in common. We have also calculated the evolutionary distances on the basis of the SSU rRNA sequences reported by Saul et al. (23) of those Thermus strains used in this study (Table 3 ) by the maximum-likelihood method described by Kishino and Hasegawa (13). The maxi- mum-likelihood method takes into account differences in rates of transitions and transversions as well as the different equilibrium frequencies of each nucleotide. As such, this is the most appropriate method for species such as Thermus with a bias towards a higher $\mathrm{G}+\mathrm{C}$ content. The maximum-likelihood method is implemented in DNADIST, a computer program that is part of J. Felsenstein's Phylogenetic Inference Package (available from J. Felsenstein, University of Washington, Seattle, Wash.). A close look at the two distance matrices shows that there is a close linear correlation between the distances estimated from PFGE-RFLP data and those from SSU rRNA sequences, particularly between those of more closely related taxa (Fig. 2). The Pearson correlation coefficient between the two sets of distances is 0.88 . Mantel's test (25) confirmed that this correlation is highly significant $(P<0.006)$.

The neighbor-joining tree obtained from PFGE-RFLP data is shown in Fig. 3. The tree obtained by Saul et al. (23), pruned to show only those taxa in common with those in this study, is also shown, and it is apparent that there is broad agreement between the two trees. Note that in the study by Saul et al. (23), while there is strong bootstrap support (5) for the divergence of Thermus strain HB27 and the strains Tok20, Tok3, W28, Rt41A, T351, and OK6, there is weak or no support for the

TABLE 2. Evolutionary distances based on PFGE-RFLPs ${ }^{a}$

\begin{tabular}{|c|c|c|c|c|c|c|c|c|c|c|c|c|c|c|}
\hline \multirow{2}{*}{ Strain } & \multicolumn{14}{|c|}{ Expected no. of nucleotide substitutions per site } \\
\hline & YVII-51B & YT-1 & T351 & T. sp. & OK6 & Rt41A & W28P & W28 & Tok21 & Tok20 & Tok3 & Fiji3 & HB27 & HB8 \\
\hline YVII-51B & 0.0000 & & & & & & & & & & & & & \\
\hline YT-1 & 0.6164 & 0.0000 & & & & & & & & & & & & \\
\hline T351 & 0.4737 & 0.3739 & 0.0000 & & & & & & & & & & & \\
\hline T. sp. & 0.6164 & 0.5079 & 0.1194 & 0.0000 & & & & & & & & & & \\
\hline OK6 & 0.6532 & 0.5546 & 0.1588 & 0.2050 & 0.0000 & & & & & & & & & \\
\hline Rt41A & 0.6352 & 0.5318 & 0.1396 & 0.1194 & 0.2246 & 0.0000 & & & & & & & & \\
\hline W28P & 0.8605 & 0.7336 & 0.4825 & 0.4555 & 0.7888 & 0.4825 & 0.0000 & & & & & & & \\
\hline W28 & 0.6352 & - & 0.2870 & 0.2658 & 0.3072 & 0.2870 & 0.7621 & 0.0000 & & & & & & \\
\hline Tok21 & 0.9592 & - & 0.8821 & 0.8605 & 0.9027 & 0.8821 & 0.8140 & 0.5967 & 0.0000 & & & & & \\
\hline Tok20 & 0.9592 & - & 0.3264 & 0.3072 & 0.3449 & 0.3264 & 0.8140 & 0.2434 & 0.3626 & 0.0000 & & & & \\
\hline Tok3 & 0.4555 & 0.7888 & 0.3739 & 0.3509 & 0.5546 & 0.3739 & 0.4555 & 0.3739 & 0.5762 & 0.4167 & 0.0000 & & & \\
\hline Fiji3 & - & - & - & - & - & - & - & - & - & - & - & 0.0000 & & \\
\hline HB27 & 0.4737 & - & 0.8379 & 0.8140 & 0.8605 & 0.8379 & - & 0.5546 & 0.5967 & 0.5967 & 0.8140 & - & 0.0000 & \\
\hline HB8 & - & - & - & - & - & - & 0.6705 & - & 0.8140 & - & - & - & - & 0.0000 \\
\hline
\end{tabular}

${ }^{a}$ The lower triangular evolutionary distance matrix used PFGE-RFLP data. - , nonestimable distances. See Materials and Methods for information on the methods used to estimate the distances. 
TABLE 3. Evolutionary distances based on SSU rRNA sequences ${ }^{a}$

\begin{tabular}{|c|c|c|c|c|c|c|c|}
\hline \multirow{2}{*}{ Strain } & \multicolumn{7}{|c|}{ Expected no. of nucleotide substitutions per site } \\
\hline & $\mathrm{T} 351$ & OK6 & Rt41A & W28 & Tok20 & Tok3 & HB27 \\
\hline T351 & 0.0000 & & & & & & \\
\hline OK6 & 0.0007 & 0.0000 & & & & & \\
\hline Rt41A & 0.0013 & 0.002 & 0.0000 & & & & \\
\hline W28 & 0.0034 & 0.0041 & 0.0048 & 0.0000 & & & \\
\hline Tok20 & 0.0041 & 0.0048 & 0.0054 & 0.0034 & 0.0000 & & \\
\hline Tok3 & 0.0054 & 0.0061 & 0.0068 & 0.0034 & 0.0013 & 0.0000 & \\
\hline HB27 & 0.0584 & 0.0592 & 0.0599 & 0.0577 & 0.0577 & 0.0562 & 0.0000 \\
\hline
\end{tabular}

a The lower triangular evolutionary distance matrix used SSU rRNA sequence data. Evolutionary distances based on SSU rRNA sequences have been calculated only for taxa used in this study. See Materials and Methods for information on the methods used to estimate the distances.

precise patterns of divergence of the last six of these strains. It appears that the lack of support is due largely to the fact that there are insufficient differences between these SSU rRNA sequences of strains to discriminate effectively between them. Any incongruence, therefore, between the PFGE-RFLP tree and the SSU rRNA tree may be an effect of chance.
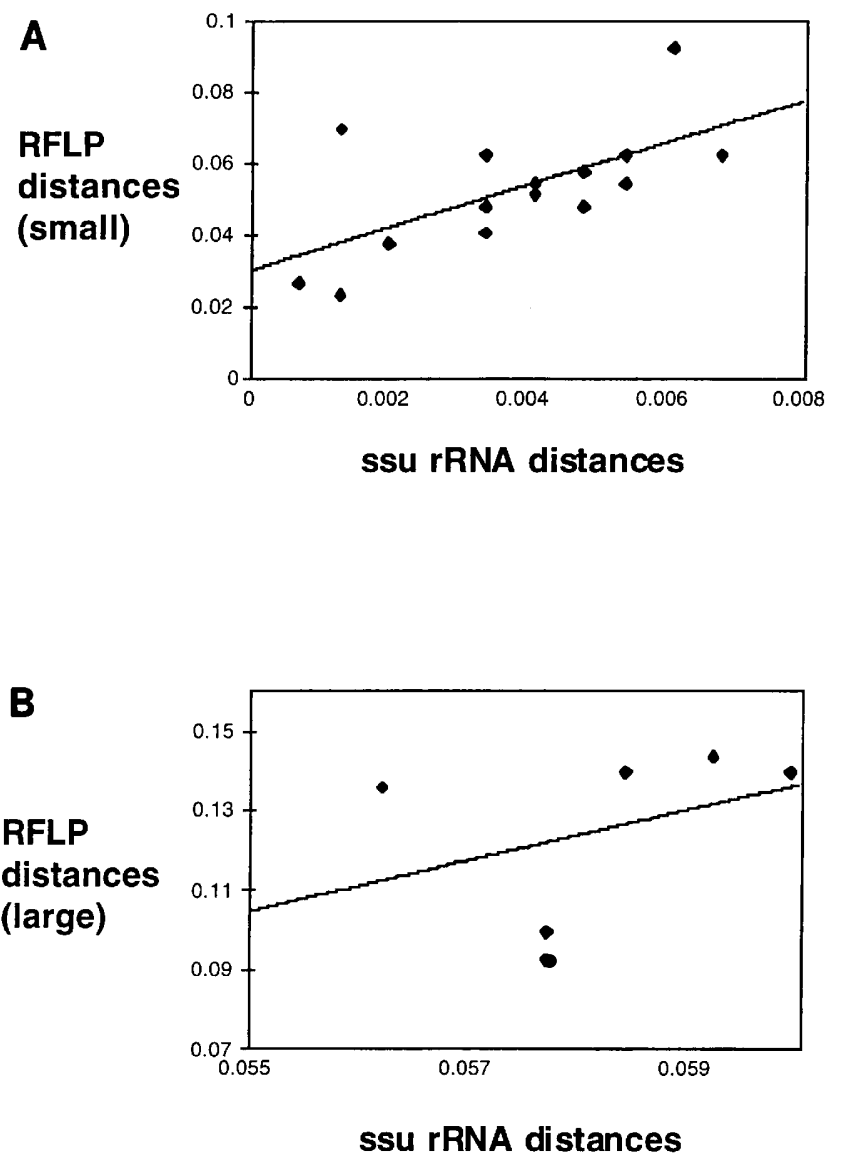

FIG. 2. Scatterplots showing relationships between evolutionary distances estimated with PFGE-RFLPs and SSU rRNA sequences. (A) Relationship between PFGE-RFLP evolutionary distances in the range 0.0 to 0.1 and SSU rRNA evolutionary distances. (B) Relationship between PFGE-RFLP evolutionary distances in the range 0.09 to 0.15 and SSU rRNA evolutionary distances. Distances are plotted in percent divergence per million years.

\section{DISCUSSION}

It is clear from our study that PFGE-RFLP profiles can successfully discriminate between different strains of Thermus, with each strain being characterized by a unique pattern of bands. Our analysis has also shown that it is also possible to use PFGE-RFLP data to construct a matrix of evolutionary distances that correlate well with those obtained from an independent data set, namely, SSU rRNAs. Therefore, it is possible to use PFGE-RFLP profiles to construct phylogenies of groups of closely related taxa. Apart from demonstrating the ability of PFGE-RFLPs to successfully recover phylogenetic information, our analysis also highlights the fact that there is an upper limit to the evolutionary distances that PFGE-RFLP profiles can be used to accurately estimate. In the simplest case, this limit is determined by the presence of at least one shared band between any two taxa. As stated above, if two taxa share no bands in common, then the evolutionary distance between the two is nonestimable. The presence or absence of shared restriction fragments amongst related taxa, in turn, is likely to be due to the expected number of sites for any given restriction enzyme along the genome and the degree of evolutionary divergence. It is reasonable to assume that the more restriction sites there are along the length of the genome, the more likely it will be for two taxa to share at least one fragment in common. Therefore, the results that we report for the upper
PFGE-RFLP

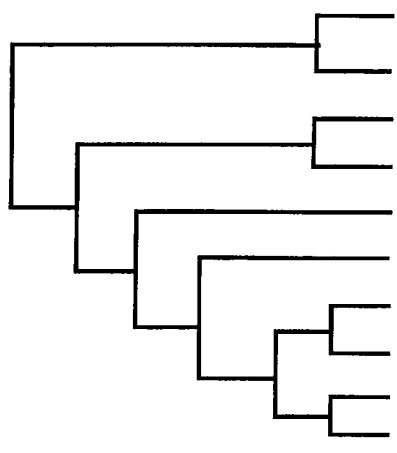

$16 \mathrm{~S}$ rRNA

YVII51B

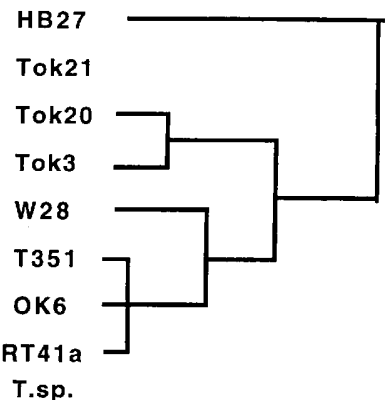

FIG. 3. Phylogenetic trees estimated with PFGE-RFLP data and SSU rRNA data. The phylogenetic tree on the left was estimated by the neighbor-joining tree-building method, with the evolutionary distances calculated between all pairs of taxa in this study that shared at least a single band in common. The phylogenetic tree on the right is a subtree of that obtained by Saul et al. (23) pruned to show only those taxa that were used in this analysis. 
limits of PFGE-RFLP evolutionary distance estimation are specific to our experimental conditions and the restriction enzyme used.

The relationship between PFGE-RFLP evolutionary distances and SSU rRNA evolutionary distances also suggests that the degree of evolutionary divergence affects the accuracy of evolutionary distance estimation. Returning to Fig. 2 and 3, it is apparent that the correlation between the larger PFGERFLP distances and SSU rRNA (correlation coefficient, $r=$ 0.36 ) is not as strong as that between the smaller PFGE-RFLP distances and SSU rRNA $(r=0.64)$. Although this result may be an artifact of different sample sizes, it is consistent with the conjecture that PFGE-RFLPs are not efficient estimators of evolutionary distance over longer periods of divergence. This observation could be due to the fact that over greater evolutionary distances, the number of bands shared between two strains may be as few as one or two. Any estimate based on one or two shared bands conceivably could increase the sampling variance of the estimate.

What may be the upper time limit to the estimation of evolutionary distances with PFGE-RFLP data? Assume that the average rate of sequence divergence between two SSU rRNA sequences is $1 \%$ per 50 million years (29) or $0.02 \%$ per million years. Note that the divergence between Thermus strain HB27 and the other strains accounts for the large PFGERFLP distances shown in Fig. 2B. Assume that these distances approach the upper limits of PFGE-RFLP distance estimation. If we estimate the time of divergence between HB27 and the other strains from the SSU rRNA evolutionary distance, we obtain an average evolutionary distance of 0.0581 or a divergence rate of $5.81 \%$; this corresponds to an expected time of divergence of 290 million years. Therefore, a divergence of about 290 million years approaches the upper limits of evolutionary distance estimation from PFGE-RFLPs under the experimental conditions we have described above. Interestingly, Hillis and Moritz (8), in their review of the appropriateness of different types of molecular data to resolve phylogenies over different time scales, have noted that restriction analysis is "marginally appropriate or appropriate under limited circumstances" with phylogenies that span 50 to 500 million years. Our value for the upper limits of PFGE-RFLP resolution falls in this range.

On the other hand, our results suggest that PFGE-RFLPs are able to resolve the evolutionary relationships of closely related taxa that would otherwise be unresolved with SSU rRNA data. It is possible that by using both PFGE-RFLPs and gene sequences a composite phylogenetic tree of the organisms in question can be constructed. We are investigating ways in which this may be done.

PFGE-RFLP data can also be used to estimate the average genomic rate of divergence if we assume that $S s p I$ restriction sites are distributed throughout the Thermus genomes. We do this by calculating the rate of sequence divergence relative to SSU rRNA. In Fig. 2, the slopes of the regression lines between PFGE-RFLP distances (taken as the dependent variable) and SSU rRNA distances (the independent variable) give values indicating how much faster the average genomic rate of divergence is relative to SSU rRNA. However, as both sets of distances are measured with error, the slope of the line is most appropriately estimated by a geometric mean regression (see reference 16 for a description of the technique). The slope of the regression line between the smaller PFGE-RFLP distances and SSU rRNA is 9.21 , whereas that of the line between the larger PFGE-RFLP distances and SSU rRNA is 17.89. The average of these two values is 13.55 , indicating that the average genomic rate of divergence is $\mathbf{1 3 . 5 5}$ times faster than that of
SSU rRNA for the Thermus genomes. Taking the SSU rRNA rate of divergence to be $0.02 \%$ per million years (see the explanation above), the average genomic rate of divergence based on our data is estimated to be $0.27 \%$ per million years (or a range of between 0.18 and $0.37 \%$ per million years, if the two different slopes obtained above are used).

We can also estimate the average genomic rate of divergence with data from the study by Ochman and Wilson (19). They estimated the rate of silent substitution of nucleotides in bacterial coding sequences at 0.7 to $0.8 \%$ per million years (midrange, $0.75 \%$ per million years) and the rate of amino acid substitution at 0.035 to $0.04 \%$ per million years (midrange, $0.0375 \%$ per million years). If we crudely assume that most of the bacterial genome is structured as coding sequences, that the majority of amino acid substitutions are the result of nucleotide substitutions in the first and second codon positions (and therefore each position has a divergence rate of $0.01875 \%$ per million years) and that all silent substitutions occur in the third codon position, then we can estimate the total rate of divergence as follows: $2 / 3 \times 0.01875+1 / 3 \times 0.75=0.2625 \%$ per million years. This value is strikingly close to our estimate of $0.27 \%$ as the average genomic rate of divergence, thus giving us greater confidence in the applicability of PFGE-RFLPs to studies of molecular evolution and phylogenies.

It appears that the PFGE-RFLP fingerprint analysis is valuable as a taxonomic aid in distinguishing between isolates of Thermus. More pertinent to bacterial systematics, perhaps, is the possibility that PFGE-RFLP data can be used to reconstruct the phylogeny of closely related strains of Thermus. We have shown that evolutionary distance models constructed with PFGE-RFLPs correlate well with those estimated by using SSU rRNA sequences. However, our analysis also suggests that there are limits to the ability of PFGE-RFLP data to estimate evolutionary distances accurately; these limits are likely to be a function of the restriction enzyme used and the degree of divergence between the taxa studied. Finally, we have shown that the estimation of the average genomic rate of nucleotide substitution using our PFGE-RFLP data agrees remarkably well with estimates derived from other studies.

\section{ACKNOWLEDGMENTS}

This work was supported by grants from the University Grants Committee, the University of Auckland Research Grants Committee, and Pacific Enzymes Ltd.

\section{REFERENCES}

1. Borges, K. M., and P. L. Bergquist. 1992. A rapid method for preparation of bacterial chromosomal DNA in agarose plugs using Thermus Rt41A proteinase. BioTechniques 12:222-223.

2. Borges, K. M., and P. L. Bergquist. 1993. Genomic restriction map of the extremely thermophilic bacterium Thermus thermophilus HB8. J. Bacteriol. 175:103-110.

3. Brock, T. D., and H. Freeze. 1969. Thermus aquaticus gen. n. and sp. n., [sic] a non-sporulating extreme thermophile. J. Bacteriol. 98:289-297.

4. Cometta, S., B. Sonnleitner, W. Sidler, and A. Fiechter. 1982. Population distribution of aerobic extremely thermophilic microorganisms in an Icelandic natural hot spring. Appl. Microbiol. Biotechnol. 16:151-156.

5. Felsenstein, J. 1985. Confidence limits on phylogenies: an approach using the bootstrap. Evolution 39:783-791.

6. Gilbert, D. A., N. Lehman, and S. J. O'Brien. 1990. Genetic fingerprinting reflects population differentiation in the Californian Channel Island fox. Nature (London) 344:764-766.

7. Grothues, D., and B. Tummler. 1987. Genome analysis of Pseudomonas aeruginosa by field inversion gel electrophoresis. FEMS Microbiol. Lett. 48:419-422.

8. Hillis, D. M., and C. Moritz. 1990. An overview of applications of 
molecular systematics, p. 502-515. In D. M. Hillis and C. Moritz (ed.), Molecular systematics. Sinauer Associates, Inc., Sunderland, Mass.

9. Howard, P. J., K. D. Harsono, and J. B. Luchansky. 1992. Differentiation of Listeria monocytogenes, Listeria innocua, Listeria ivanovii, and Listeria seeligeri by pulsed-field gel electrophoresis. Appl. Environ. Microbiol. 58:709-712.

10. Hudson, J. A., H. W. Morgan, and R. M. Daniel. 1986. A numerical classification of some Thermus isolates. J. Gen. Microbiol. 132: 531-540.

11. Hudson, J. A., H. W. Morgan, and R. M. Daniel. 1987. Thermus filiformis sp. nov., a filamentous caldoactive bacterium. Int. J. Syst. Bacteriol. 37:431-436.

12. Hudson, J. A., H. W. Morgan, and R. M. Daniel. 1989. Numerical classification of Thermus isolates from globally distributed hot springs. Syst. Appl. Microbiol. 11:250-256.

13. Kishino, H., and M. Hasegawa. 1989. Evaluation of the maximum likelihood estimate of the evolutionary tree topologies from DNA sequence data, and the branching order of the Hominoidea. J. Mol. Evol. 29:170-179.

14. Le Bourgeois, P., M. Mata, and P. Ritzenthaler. 1989. Genome comparison of Lactococcus strains by pulsed-field gel electrophoresis. FEMS Microbiol. Lett. 59:65-70.

15. Loginova, L. G., L. A. Egorova, L. A. Golovacheva, and L. M. Seregina. 1984. Thermus ruber sp. nov., nom. rev. Int. J. Syst. Bacteriol. 34:498-499.

16. McArdle, B. H. 1988. Structural relationship: regression in biology. Can. J. Zool. 66:2329-2339.

17. Nei, M. 1991. Relative efficiencies of different tree-making methods for molecular data, p. 90-128. In M. Miyamoto and J. Cracraft (ed.), Phylogenetic analysis of DNA sequences. Oxford University Press, New York.

18. Nei, M., and W.-H. Li. 1979. Mathematical model for studying genetic variation in terms of restriction endonucleases. Proc. Natl. Acad. Sci. USA 76:5269-5273.

19. Ochman, H., and A. C. Wilson. 1987. Evolution in bacteria: evidence for a universal substitution rate. 26:74-86.

20. Oshima, T., and K. Imahori. 1974. Description of Thermus thermophilus (Yoshida and Oshima) comb. nov., a nonsporulating thermophilic bacterium from a Japanese thermal spa. Int. J. Syst. Bacteriol. 24:102-112.

21. Saitou, N., and M. Nei. 1987. The neighbour-joining method: a new method for reconstructing phylogenetic trees. Mol. Biol. Evol. 4:406-425.

22. Santos, M. A., R. A. D. Williams, and M. S. da Costa. 1989. Numerical taxonomy of Thermus isolates from hot springs in Portugal. Syst. Appl. Microbiol. 12:310-315.

23. Saul, D. J., A. G. Rodrigo, R. A. Reeves, L. C. Williams, K. M. Borges, H. W. Morgan, and P. L. Bergquist. 1993. Phylogeny of twenty Thermus isolates constructed from 16S rRNA gene sequence data. Int. J. Syst. Bacteriol. 43:754-760.

24. Smith, C. L., S. R. Klco, and C. R. Cantor. 1988. Pulsed-field electrophoresis and the technology of large DNA molecules, p. 41-72. In K. Davies (ed.), Genome analysis. IRL Press, Ltd., Oxford.

25. Smouse, P. E., J. C. Long, and R. R. Sokal. 1986. Multiple regression and correlation extensions of the Mantel's test of matrix correspondence. Syst. Zool. 35:627-632.

26. Swofford, D. L., and G. J. Olsen. 1990. Phylogenetic reconstruction, p. 411-501. In D. M. Hillis and C. Moritz (ed.), Molecular systematics. Sinauer Associates, Inc., Sunderland, Mass.

27. Tanskanen, E. I., D. L. Tulloch, A. J. Hillier, and B. E. Davidson. 1990. Pulsed-field gel electrophoresis of SmaI digests of lactococcal genomic DNA, a novel method of strain identification. Appl. Environ. Microbiol. 56:3105-3111.

28. Williams, R. A. D. 1989. Biochemical taxonomy of the genus Thermus, p. 82-997. In M. S. da Costa, J.-C. Duarte, and R. A. D. Williams (ed.), Microbiology of extreme environments and its potential for biotechnology. Elsevier, London.

29. Wilson, A. C., H. Ochman, and E. M. Prager. 1987. Molecular time scale for evolution. Trends Genet. 3:241-247. 